\title{
Driven Colloidal Mixtures and Colloidal Liquid Crystals
}

\author{
H. Löwen, H. H. Wensink, and M. Rex \\ Institut für Theoretische Physik II: Weiche Materie, Heinrich-Heine-Universität Düsseldorf, \\ Universitätsstraße 1, D-40225 Düsseldorf, Germany
}

\begin{abstract}
Colloids driven by an external field are ideal model systems for non-equilibrium problems. In particular, a binary driven system driven by a constant external force exhibits a non-equilibrium phase transition towards lane formation. In oppositely charged colloidal mixtures there are several intermediate states with lateral crystalline order. Driving tracer particles through quiescent liquid crystals generates rhythmic clustering at high drives. Driven binary mixtures of liquid crystals and spheres exhibit again lane formation of pure phases containing particles driven alike with a local biaxiality at the driven interface between pure spheres and pure rods. The results presented are based on Brownian dynamics computer simulation and theory.
\end{abstract}

Keywords: Colloids, Driven systems, Lane Formation, Liquid crystals, Non-equilibrium

PACS: $82.70 . \mathrm{Dd}, 05.40 . \mathrm{-a}, 61.20 . \mathrm{Ja}$

\section{INTRODUCTION}

Colloidal suspensions are excellent model systems for equilibrium and non-equilibrium situations. In particular, the recent development of well-characterized particles and their control by external fields like optical tweezers have it made possible to expose colloids to controlled and tailored non-equilibrium conditions [1]. One possible set-up consists of dragging a tracer particle through a quiescent suspension which will destroy the colloidal order along its trace. The driving speed can be imposed by the external force field acting on the tagged bead particle. Another possibility is to consider a binary mixture of oppositely driven particles. This can e.g. be realized by using oppositely charged particles in an electric field [2] or by a combination of gravity and magnetic response [3].

In this paper we review recent progress in this field and concentrate mainly on results obtained by Brownian dynamics computer simulations. First we discuss a binary mixtures with identical interactions, the different species just interact differently with the external field imposed. The formation of lanes is discussed. Then we turn to oppositely charged particle where the like-species interact repulsively while the cross-interaction between different species is attractive. We subsequently consider colloidal liquid crystals. Dragging a spherical tracer particle through a nematic suspension perpendicular to the director will give rise to rhythmic clustering of rods in front of the moving particle. Finally we expose a binary mixture of rods and spheres to a constant driving force which acts differently on the two species and thus we obtain again lanes of rods and spheres driven alike. At the interface there is a further alignment which can be traced back to an equilibrium effect under confinement.

\section{DRIVEN BINARY MIXTURES OF SPHERICAL PARTICLES WITH REPULSIVE CROSS-INTERACTION}

In this case, the symmetric Yukawa-interaction has been studied in great detail both in two and three spatial dimensions [5]. In this case, without driving field, all particles interact exactly via the same interparticle potential. For increasing driving force, the system goes from a steady state with anisotropic correlations towards a state where macroscopic lanes are formed. The transition possesses a significant hysteresis, hence it can be classified as a first-order non-equilibrium phase transition. For very long times the system tends to exhibit macroscopic phase separation where two blocks of particles are sliding against each other, each block containing particles driven alike.

There is a reentrance effect for increasing density at fixed driving force: at small densities, there is no lane formation, at intermediate densities lanes are formed and at very high densities jammed configurations dominate which destroy again the lanes [6]. A dynamical density functional theory [7] describes the trends quantitatively well as compared to the Brownian dynamics computer simulations data [6]. These behavior is reminiscent to pedestrian dynamics [8].

The scenario described above is stable for asymmetric Yukawa interactions [9]. It occurs also for low temperatures where the starting state is a mixed binary crystal [10]. Finally we remark that it is irrelevant whether both species are driven or only one $[5,11]$. If the two driving forces acting on the two particle species are tilted relative to each other, there is again lane formation but with tilted lanes [10]. A very strong repulsive cross-

\footnotetext{
CP982, Complex Systems, $5^{\text {th }}$ International Workshop on Complex Systems

edited by $\mathrm{M}$. Tokuyama, I. Oppenheim, and H. Nishiyama

(C) 2008 American Institute of Physics 978-0-7354-0501-1/08/\$23.00
} 


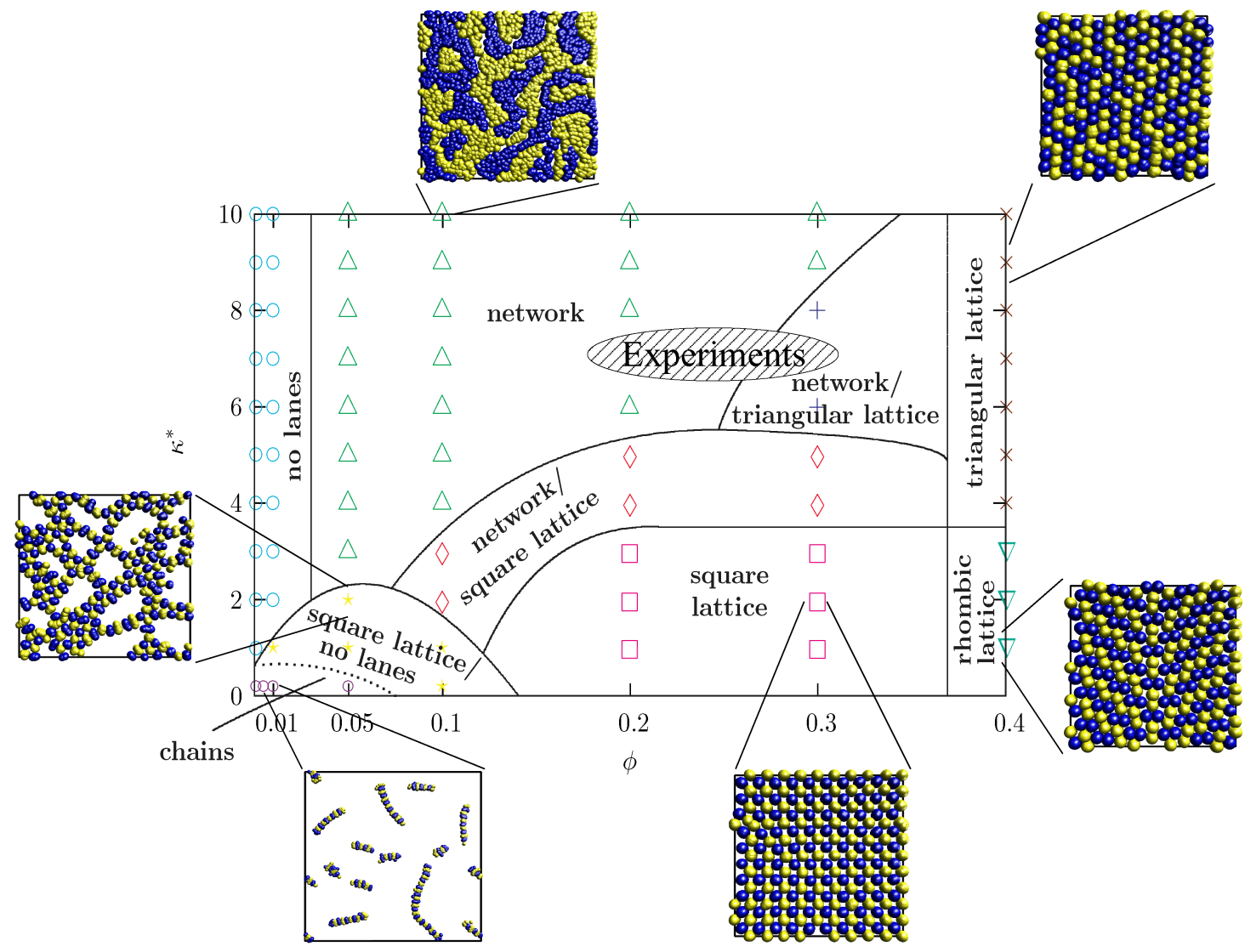

FIGURE 1. Non-equilibrium steady-state phase diagram for a constant driving force. A simulation snapshot of the projection of the particle coordinates perpendicular to the driving field is displayed for each different state. We find a variety of different phases involving lane chains at small volume fraction and low screening and lanes with two-dimensional crystalline order perpendicular to the field at high volume fraction. The lateral crystalline order can be a square, triangular or rhombic lattice. The hatched region indicates the parameters for which experimental results are published by Leunissen et al. [2]. For more details we refer to Ref. [4].

interaction leads to macro-phase separation. The time evolution of phase separation from a initially mixed state has been studied under an external drive in Ref. [12]. The Rayleigh-Taylor-like instability at a fluid-fluid interface, on the other hand, has been considered in Ref. [13].

\section{DRIVEN BINARY MIXTURES OF SPHERICAL PARTICLES WITH ATTRACTIVE CROSS-INTERACTION}

Recent experiments on oppositely charged particles in an electric field [2] have motivated studies of a Yukawa model with attractive cross-interaction. Using Brownian dynamics computer simulations in three dimensions, a wealth of different lateral structures were found resulting from a competition between Coulomb interaction and friction and sliding lanes [4]. The steady states involve lateral network-like structures and square, triangular and rhombic lattices of oppositely driven lanes. In two spatial dimensions, on the other hand, there is just an alternating stripe formation [14].

The steady state phase diagram is summarized in Figure 1 [4]. Here a fixed external force is used and the results are shown for different screening parameters $\kappa^{*}$ and volume fractions $\phi$. Here, the interaction is a superposition of a (softened) core of diameter $\sigma$ and a Yukawa interaction $\left.\pm U_{0} \exp \left(-\kappa^{*} r / \sigma\right) / r\right)$. The parameters are: $U_{0} / \sigma k_{B} T=50, F_{0} \sigma / k_{B} T=236$, where $k_{B} T$ denotes the thermal energy and $F_{0}$ is the external driving forces induced by the electric field.

The network structure was also found in real space experiments of driven oppositely charged colloids [2]. This is confirmed by simulation by using estimated model parameters suitable to describe the experimental situation, see the star symbol in Figure 1. Still a comparison in the full parameter space has to be performed. Furthermore a theory, e.g. in the spirit of dynamical density functional theory $[6,7]$ is still missing in this case. 

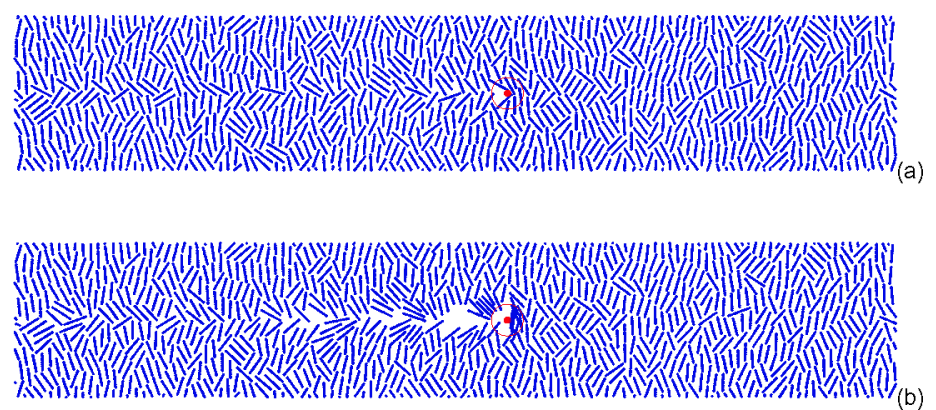

$\lambda_{0}$
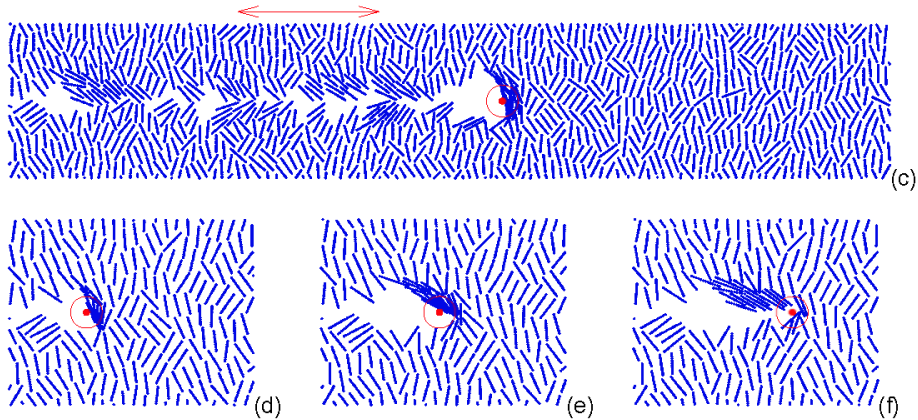

FIGURE 2. Snapshots of the nematic structure at (a) $v^{*}=100$, (b) $v^{*}=1000$ and (c) $v^{*}=4000$. The driven sphere is indicated by the encircled dot. The sequence (d)-(f) depicts the growth and rotation of a single rod cluster at $v^{*}=4000$. The arrow indicates a cluster-cluster correlation length $\lambda_{0}=5 L_{0}$. Figure taken from Ref. [15].

\section{DRIVING A SPHERE THROUGH A NEMATIC COLLOIDAL CRYSTAL}

The Brownian dynamics can readily be generalized to rod-like particles $[16,17]$. Then two translational diffusion constants, parallel and perpendicular to the orientation of the particle have to be considered as well as on orientational diffusion constant. For large rod aspect ratios, the suspensions can form a nematic state where the director is oriented.

Recently an interesting rhythmic clustering behavior was found in a driven system [15] with Yukawa resp. Yukawa segment interactions. In particular, a sphere was dragged by constant speed through a two-dimensional nematic crystal. The driving direction was perpendicular to the nematic director. At high driving strengths, the driven sphere generates clusters of rods which rhythmically grow and dissolve with a characteristic frequency. This corresponds to a characteristic length along the trace of the driven sphere. The simulation data are in accordance with a dynamical scaling theory. A typical simulation snapshot is shown in Figure 2.

The parameters are $U_{0} / \sigma k_{B} T=0.065$ for the segment-segment interaction, $\sigma / L_{0}=16.7$ is the ratio of the rod length $L_{0}$ and diameter $\sigma$ and $\kappa^{*} \sigma=0.36$. We simulated $N=900$ rods each composed of 13 segments. The interaction between the tracer sphere and the rods is of the form $1 / r^{12}$ and the amplitude is tuned such that the sphere cannot penetrate the rods. Furthermore, $v^{*}=v L_{0} / D_{0}^{T} \gg 1$ is the speed of the dragged tracer sphere expressed in units of the typical translational diffusion speed via $D_{0}^{T}$, the average translation diffusion coefficient of the rod. The dimensionless density of the systems is $N L_{0}^{2} / A=3.0$ ( $A$ is the area of the rectangular simulation box) which is high enough to ensure a $2 \mathrm{D}$ bulk nematic configuration with a director pointing along the vertical axis of the box.

Clearly a cluster of rods is formed in front of the driven particles which dissolves and leaves a clustered structure in the "wake" of the driven sphere. Still an experimental verification of this effect is missing but it is in principle possible to prepare nematic colloidal liquid crystals and drag tracer particles through them.

\section{DRIVEN BINARY MIXTURES OF REPULSIVE SPHERES AND RODS}

Finally one may consider a binary mixtures of spheres and rods and drive the rods by a constant force acting on their center of mass. We have performed Brownian dynamics computer simulations in two spatial dimensions by using a repulsive Yukawa interaction, similar to that in Ref. [15]. In detail, the segment-segment interactions and 

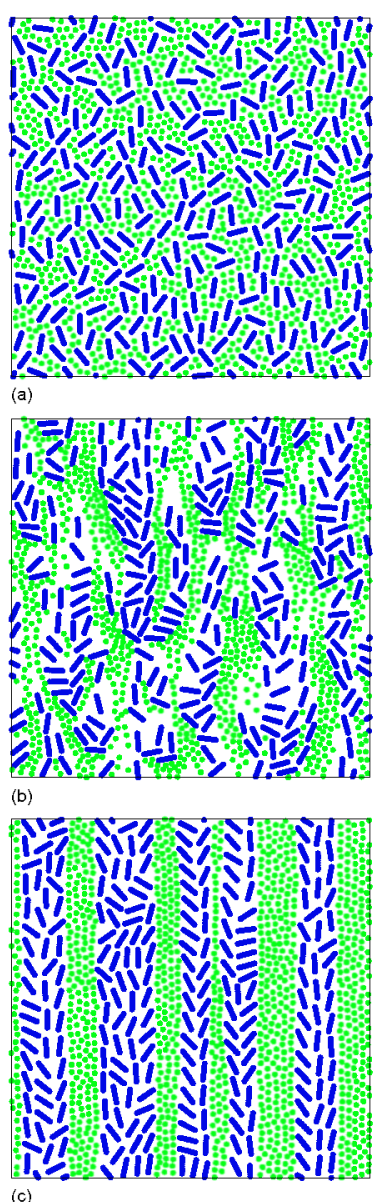

(c)

FIGURE 3. Snapshots of the driven rod-sphere mixture at constant driving force. (a) Equilibrium state at $t=0$. (b) Intermediate driven state at $t=1.4$. (c) Steady-state laning structure at $t=45$. The direction of the applied force is indicated by the arrow. The time $t$ is measured in units of the typical Brownian translational relaxation time of a single rod.

the rod aspect-ratio are the same as described in the previous paragraph. Here, the cross interaction between the spheres and the rod segment is identical to the segmentsegment interaction of the rods. Furthermore, we have $N_{\text {rods }} / N_{\text {spheres }}=0.25$ with a total of 1250 particles using 10 Yukawa segments for each rod. The driving force acting on both species is fixed at $F_{0} L_{0} / k_{B} T=100$. Note that the rod density is smaller than in the previous case such as to guarantee a thermodynamically stable reference mixture with an isotropic rod configuration.

In this system, sufficiently strong drives lead again to lane formation similar to that discussed in the 2nd paragraph for spheres. However, there is an additional aligning effect in the lanes of the driven rods. The lanes slide against each other and at this sliding interface a homeotropic ordering of the rods is observed. This means that the rods are oriented perpendicular to (or make a typical angle with) this interface which can clearly been seen from the simulation snapshots shown in Figure 3. This effect can be explained from a static concept and stems from a subtle interplay between and external potential imposed by the quasi-static soft walls composed of the spheres and the orientation-dependent pair potential of the rods. Moreover, upon averaging over the rod orientations we find that the local orientational structure within the lanes is in fact biaxial. This effect is also observed in strongly confined rods in equilibrium, see e.g. [18]. In conclusion, the drive here produces a self-organized confinement and confinement-induced effects, such as tilting and biaxiality, of the rods take place.

\section{CONCLUSIONS}

Colloidal suspensions which are driven by an external field exhibit interesting non-equilibrium phase transitions such as laning, rhythmic clustering and selforganized confinement. In principle the results of Brownian dynamics computer simulations can be checked by real-space experiments but they serve also as benchmark data to test theories such as the dynamical density functional theory.

There is still a need to understand the influence of hydrodynamic interactions mediated by the solvent. Fortunately the leading long-ranged contribution is screened for electrophoresis [19], therefore one might hope that they are not relevant for charged colloids in an electric field while it seems that they are more important for sedimentation [20]. Certainly more work is needed in this direction following more sophisticated simulation schemes, e.g. as proposed by Yamamoto and coworkers [21].

Finally it would be very interesting to study the lane formation in a confined geometry between parallel plates. It was found that jammed configurations are significantly stabilized by confinement relative to lane formation [22]. More simulations to study systematically the effect of confinement are needed. It would also be interesting to perform an instability analysis based on dynamical density functional theory for the confined case following the strategy put forward in Ref. [7].

\section{ACKNOWLEDGMENTS}

We thank A. van Blaaderen, M. Leunissen, D. Cleaver and R. Yamamoto for helpful discussions. This work was financially supported by the DFG under SFB-TR6 (project section D1). 


\section{REFERENCES}

1. H. Löwen, J. Phys.: Condens. Matter 13, R415-423 (2001).

2. M. E. Leunissen, C. G. Christova, A. P. Hynninen, C. P. Royall, A. I. Campbell, A. Imhof, R. van Roij, and A. van Blaaderen, Nature 437, 235-240 (2005).

3. M. Köppl, P. Henseler, A. Erbe, P. Nielaba, and P. Leiderer, Phys. Rev. Lett. 97, 208302-1-4 (2006).

4. M. Rex, and H. Löwen, Phys. Rev. E 75, 051402-1-10 (2007).

5. J. Dzubiella, G. P. Hoffmann, and H. Löwen, Phys. Rev. E 65, 021402-1-8 (2002).

6. J. Chakrabarti, J. Dzubiella, and H. Löwen, Phys. Rev. E 70, 012401-1-4 (2004).

7. J. Chakrabarti, J. Dzubiella, and H. Löwen, Europhys. Lett. 61, 415-421 (2003).

8. R. Jiang, D. Helbing, P. K. Shukla, and Q. S. Wu, Physica A 368, 567-574 (2006).

9. H. Löwen, and J. Dzubiella, Faraday Dicussions 123, 99-105 (2003).

10. J. Dzubiella, and H. Löwen, J. Phys.: Condens. Matter 14, 9383-9395 (2002).

11. C. Reichhardt, and C. J. Olson-Reichhardt, Phys. Rev. E 74, 011403-1-7 (2006).

12. H. Löwen, C. N. Likos, R. Blaak, S. Auer, V. Froltsov, J. Dzubiella, A. Wysocki, and H. M. Harreis, "Colloidal suspensions driven by external fields," in AIP Conference Proceedings, edited by M. Tokuyama, and I. Oppenheim, 2004, vol. 708, pp. 3-7.

13. A. Wysocki, and H. Löwen, J. Phys.: Condens. Matter 16, $7209-7224$ (2004).

14. R. R. Netz, Europhys. Lett. 63, 616-622 (2003).

15. H. H. Wensink, and H. Löwen, Phys. Rev. Lett. 97 , 038303-1-4 (2006).

16. H. Löwen, Phys. Rev. E 50, 1232-1242 (1994).

17. T. Kirchhoff, H. Löwen, and R. Klein, Phys. Rev. E 53, 5011-5022 (1996).

18. A. Chrzanowska, P. I. C. Teixeira, H. Ehrentraut, and D. J. Cleaver, J. Phys.: Condens. Matter 13, 4715-4726 (2001).

19. D. Long, and A. Ajdari, Eur. Phys. J. E 4, 29-32 (2001).

20. C. P. Royall, J. Dzubiella, M. Schmidt, and A. van Blaaderen, Phys. Rev. Lett. 98, 188304-1-4 (2007).

21. K. Kim, Y. Nakayama, and R. Yamamoto, Phys. Rev. Lett. 96, 208302-1-4 (2006).

22. D. Helbing, I. J. Farkas, and T. Viscek, Phys. Rev. Lett. 84, 1240-1243 (2000). 